\title{
NON-HOMOGENEOUS COMBINATIONS OF COEFFICIENTS OF UNIVALENT FUNCTIONS
}

\author{
R. A. KORTRAM and O. TAMMI'
}

\section{Introduction}

G. Schober communicated us in 1978 the following problem: Determine $\max _{f \in S} \operatorname{Re}\left(a_{3}+i a_{2}\right)$ (see also [4], p. 84). In this paper we consider the general problem of finding max $\operatorname{Re}\left(a_{3}+\lambda a_{2}\right)$ for an arbitrary complex parameter $\lambda$ and for functions $f \in S(b)$. Löwner's parametric method shall be extensively used in the following considerations.

\section{The case of $S_{R}(b)$}

Let $b \in(0,1)$ and let $\Delta=\{z|| z \mid<1\}$. The class $S_{R}(b)$ consists of the univalent functions $f: \Delta \rightarrow \Delta$ for which $f(z)=b\left\{z+a_{2} z^{2}+a_{3} z^{3}+\ldots\right\}$ with $a_{k} \in R$. The problem reduces to the study of $a_{3}+\lambda a_{2}$ for $\lambda \in R$. In [2] pp. 8, 9, 10 we have derived the following sharp estimates for functions $f \in S_{R}(b)$ :

$$
\begin{aligned}
& a_{3} \geqq a_{2}^{2}-\left(1-b^{2}\right), \\
& a_{3} \leqq 1-b^{2}+a_{2}^{2}\left(1+\frac{1}{\log b}\right) \text { if }\left|a_{2}\right| \leqq-2 b \log b, \\
& a_{3} \leqq a_{2}^{2}+1-b^{2}-2\left(\sigma^{2}-b^{2}\right)+4 \sigma^{2} \log \sigma \quad \text { if } \quad\left|a_{2}\right|>-2 b \log b .
\end{aligned}
$$

The parameter $\sigma$ is determined by $\sigma-\sigma \log \sigma=b+\left|a_{2}\right| / 2$.

Taking into account that $\left|a_{2}\right| \leqq 2(1-b)$ we immediately obtain

$$
\min \left(a_{3}+\lambda a_{2}\right)=\left\{\begin{array}{l}
-\left(1-b^{2}\right)-\frac{1}{4} \lambda^{2} \text { if }|\lambda| \leqq 4(1-b), \\
3-8 b+5 b^{2}-2(1-b)|\lambda| \text { if }|\lambda|>4(1-b) .
\end{array}\right.
$$

1) This work was supported in part by a research grant from the Katholieke Universiteit Nijmegen. 
The upper bound is more complicated. We have to distinguish between the following two cases:

1) $\left.1+\frac{1}{\log b} \leqq 0, \quad 2\right) \quad 1+\frac{1}{\log b}>0$.

In both these cases we have to deal separately with the possibilities $\left|a_{2}\right| \leqq-2 b \log b$ and $\left|a_{2}\right|>-2 b \log b$. After an elementary but rather long calculation we arrive at the following result.

Case 1: $e^{-1} \leqq b<1$.

$$
\max \left(a_{3}+\lambda a_{2}\right)=\left\{\begin{array}{l}
1-b^{2}-\frac{1}{4} \lambda^{2} \frac{\log b}{1+\log b} \quad \text { if } \quad|\lambda| \leqq 4 b(1+\log b), \\
1-b^{2}+|\lambda|\left(\sigma-\frac{1}{4}|\lambda|\right)+2(\sigma-b)^{2} \quad \text { if } \quad 4 b(1+\log b)<|\lambda|<4 b, \\
3-8 b+5 b^{2}+2(1-b)|\lambda| \text { if }|\lambda| \geqq 4 b .
\end{array}\right.
$$

The number $\sigma \in[b, 1]$ is determined by $\sigma \log \sigma+b=|\lambda| / 4$.

Case 2: $0<b<e^{-1}$.

$$
\max \left(a_{3}+\lambda a_{2}\right)=\left\{\begin{array}{l}
1-b^{2}+|\lambda|\left(\sigma-\frac{1}{4}|\lambda|\right)+2(\sigma-b)^{2} \quad \text { if } \quad|\lambda|<4 b, \\
3-8 b+5 b^{2}+2(1-b)|\lambda| \text { if }|\lambda| \geqq 4 b .
\end{array}\right.
$$

The number $\sigma \in[b, 1]$ is determined by $\sigma \log \sigma+b=|\lambda| / 4$.

\section{The general case $S(b)$}

As usual, for $b \in(0,1), S(b)$ consists of the univalent functions $f: \Delta \rightarrow \Delta$ for which $f(z)=b\left\{z+a_{2} z^{2}+a_{3} z^{3}+\ldots\right\}$. Instead of $S$ we shall sometimes write $S(0)$. We shall consider the dense subclass of slit-functions. For these the following Löwner expressions hold:

$$
a_{2}=-2 \int_{b}^{1} x(u) d u, \quad a_{3}=a_{2}^{2}-2 \int_{b}^{1} u \varkappa^{2}(u) d u,
$$

where $\chi(u)=e^{i \vartheta(u)}$ is a continuous function. For a piecewise continuous $\vartheta$ the formulae (1) still define coefficients of functions $f \in S(b), b \in[0,1)$.

For a given number

we have

$$
\lambda=\mu+i v
$$

(2a) $\operatorname{Re}\left(a_{3}+\lambda a_{2}\right)=4\left(\int_{b}^{1} \cos \vartheta(u) d u\right)^{2}-4\left(\int_{b}^{1} \sin \vartheta(u) d u\right)^{2}-2 \int_{b}^{1} u \cos 2 \vartheta(u) d u$

$$
-2 \mu \int_{b} \cos \vartheta(u) d u+2 v \int_{b}^{1} \sin \vartheta(u) d u
$$


Consider first the case $v=0$, i.e. $\lambda \in R$. In this case we have

$$
\operatorname{Re}\left(a_{3}+\mu a_{2}\right) \leqq 4\left(\int_{b}^{1} \cos \vartheta(u) d u\right)^{2}-2 \int_{b}^{1} u \cos 2 \vartheta(u) d u-2 \mu \int_{b}^{1} \cos \vartheta(u) d u
$$

For the Löwner functions $f \in S_{R}(b)$ we have, according to [5] p. 10,

$$
a_{2}=-2 \int_{b}^{1} \cos \vartheta(u) d u, \quad a_{3}=a_{2}^{2}-2 \int_{b}^{1} u \cos 2 \vartheta(u) d u .
$$

Thus in this case the maximum is attained in the subclass $S_{R}(b)$, for which the solution was determined above.

From now on we assume that

$$
v \neq 0 \text {. }
$$

By considering $\overline{f(\bar{z})}$ instead of $f(z)$ we see that $v \int_{b}^{1} \sin \vartheta(u) d u \geqq 0$ in the maximum case. Similarly, by considering $-f(-z)$ instead of $f(z)$ we find $\mu \int_{b}^{1} \cos \vartheta(u) d u \leqq 0$ in the maximum case. For brevity, let us normalize

$$
v>0, \quad \mu \leqq 0 ; \quad \int_{b}^{1} \sin \vartheta(u) d u \geqq 0, \quad \int_{b}^{1} \cos \vartheta(u) d u \geqq 0 .
$$

Rewriting (2a) we obtain

$$
\begin{gathered}
\operatorname{Re}\left(a_{3}+\lambda a_{2}\right)=1-b^{2}+4\left(\int_{b}^{1} \cos \vartheta(u) d u\right)^{2}-4\left(\int_{b}^{1} \sin \vartheta(u) d u\right)^{2} \\
-4 \int_{b}^{1} u \cos ^{2} \vartheta(u) d u-2 \mu \int_{b}^{1} \cos \vartheta(u) d u+2 v \int_{b}^{1} \sin \vartheta(u) d u .
\end{gathered}
$$

Let us replace the maximizing $\vartheta$ by $\tilde{\vartheta}$ which is obtained from $\vartheta$ by changing $\vartheta$ into $\pi-\vartheta$ on an arbitrary subinterval $l$ of $[b, 1]$. The functional $(2)$ is then altered in such a way that

$$
\begin{gathered}
\operatorname{Re}\left(a_{3}+\lambda a_{2}\right)-\operatorname{Re}\left(\tilde{a}_{3}+\lambda \tilde{a}_{2}\right) \\
=16\left(\int_{b}^{1} \cos \vartheta(u) d u-\frac{\mu}{4}\right) \int_{l} \cos \vartheta(u) d u-16\left(\int_{l} \cos \vartheta(u) d u\right)^{2} .
\end{gathered}
$$

We deduce from this that if $\cos \vartheta(u) \neq 0$ at some point, then $\cos \vartheta(u)>0$, i.e.

in the maximum case.

$$
\cos \vartheta(u) \geqq 0
$$

Similarly, we can deduce from (2) that in the maximum case $\cos \vartheta$ is decreasing (and hence $|\sin \vartheta|$ is increasing), since the only part depending on the arrangement of the values of $\cos \vartheta$ is $-\int_{b}^{1} u \cos ^{2} \vartheta(u) d u$.

The part in (2) which depends explicitly on $\sin \vartheta$ is

$$
-4\left(\int_{b}^{1} \sin \vartheta(u) d u\right)^{2}+2 v \int_{b}^{1} \sin \vartheta(u) d u .
$$


If $v \geqq 4(1-b)$, it follows from (2) that in the maximum case

$$
\sin \vartheta(u) \geqq 0 .
$$

For, if $\sin \vartheta$ assumed negative values, we could change its sign without affecting $\cos \vartheta$ and thus increase $\int_{b}^{1} \sin \vartheta(u) d u$, and by doing so, we would increase the above mentioned part determined by $\sin \vartheta$. - Therefore, if in the maximum case $\sin \vartheta$ assumes negative values, we must have $v<4(1-b)$ and $\int_{b}^{1} \sin \vartheta(u) d u=v / 4$.

A necessary condition for the function $\vartheta$ to be extremal is that the first order variation of (2) is zero. This leads to the condition

$\left(\int_{b}^{1} \cos \vartheta(u) d u-\frac{1}{4} \mu\right) \sin \vartheta(u)+\left(\int_{b}^{1} \sin \vartheta(u) d u-\frac{1}{4} v\right) \cos \vartheta(u)=u \sin \vartheta(u) \cos \vartheta(u)$.

If $\vartheta$ has to give rise to the maximum, then the second order variation has to be nonpositive. This leads to the following condition: For all piecewise continuous functions $\varphi$ we have

$$
\begin{aligned}
& \left(\int_{b}^{1} \varphi(u) \sin \vartheta(u) d u\right)^{2}-\left(\int_{b}^{1} \varphi(u) \cos \vartheta(u) d u\right)^{2} \\
& +\left(\int_{b}^{1} \sin \vartheta(u) d u-\frac{1}{4} v\right) \int_{b}^{1} \varphi^{2}(u) \sin \vartheta(u) d u \\
& -\left(\int_{b}^{1} \cos \vartheta(u) d u-\frac{1}{4} \mu\right) \int_{b}^{1} \varphi^{2}(u) \cos \vartheta(u) d u \\
& +\int_{b}^{1} u \varphi^{2}(u) \cos ^{2} \vartheta(u) d u-\int_{b}^{1} u \varphi^{2}(u) \sin ^{2} \vartheta(u) d u \leqq 0 .
\end{aligned}
$$

\section{The perfect square representation}

Let $C$ be an arbitrary parameter. The identity

$$
\begin{gathered}
a_{3}-a_{2}^{2}-C a_{2}+\frac{1}{2} C^{2} \log b=-2 \int_{b}^{1} A^{2}(u) d u ; \\
A(u)=\sqrt{u}\left(\varkappa(u)-\frac{C}{2 u}\right)
\end{gathered}
$$

follows from the formulae (1). Hence

$$
\begin{aligned}
& \operatorname{Re}\left(a_{3}-a_{2}^{2}-C a_{2}+\frac{1}{2} C^{2} \log b\right) \\
& =-2 \int_{b}^{1} \operatorname{Re} A^{2}(u) d u=2 \int_{b}^{1}|A(u)|^{2} d u-4 \int_{b}^{1}(\operatorname{Re} A(u))^{2} d u \\
& =1-b^{2}-\frac{1}{2}|C|^{2} \log b+\operatorname{Re}\left(\bar{C} a_{2}\right)-4 \int_{b}^{1}(\operatorname{Re} A(u))^{2} d u .
\end{aligned}
$$


Let us make use of the choice

which gives

$$
C=-\left(a_{2}+\frac{1}{2} \lambda\right)
$$

$$
\begin{aligned}
& \operatorname{Re}\left(a_{3}+\lambda a_{2}\right)=1-b^{2}+\frac{1}{4} v^{2}-\frac{1}{4} \mu^{2} \log b-\left(\operatorname{Im} a_{2}+\frac{1}{2} v\right)^{2} \\
& -(1+\log b)\left(\operatorname{Re} a_{2}\right)^{2}-\mu \operatorname{Re} a_{2} \log b-4 \int_{b}^{1}(\operatorname{Re} A(u))^{2} d u
\end{aligned}
$$

If $b \neq e^{-1}$, this can be written as

$$
\begin{aligned}
& \operatorname{Re}\left(a_{3}+\lambda a_{2}\right)=1-b^{2}+\frac{1}{4} v^{2}-\frac{1}{4} \mu^{2} \frac{\log b}{1+\log b}-\left(\operatorname{Im} a_{2}+\frac{1}{2} v\right)^{2} \\
& -(1+\log b)\left(\operatorname{Re} a_{2}+\frac{\mu \log b}{2(1+\log b)}\right)^{2}-4 \int_{b}^{1}(\operatorname{Re} A(u))^{2} d u .
\end{aligned}
$$

We can also rewrite (4) in the form

$$
\begin{aligned}
& \operatorname{Re}\left(a_{3}+\lambda a_{2}\right)=1-b^{2}+\frac{1}{4} v^{2}-\left(\operatorname{Re} a_{2}\right)^{2}-\left(\operatorname{Re} a_{2}+\frac{1}{2} \mu\right)^{2} \log b \\
& -\left(\operatorname{Im} a_{2}+\frac{1}{2} v\right)^{2}-4 \int_{b}^{1}(\operatorname{Re} A(u))^{2} d u .
\end{aligned}
$$

The representation (4) is closely related to those used by Haario and Jokinen in [1].

\section{Extremals of type $2: 2$}

Suppose that $e^{-1}<b<1$ (hence $1+\log b>0$ ) and obtain from (4a)

$$
\operatorname{Re}\left(a_{3}+\lambda a_{2}\right) \leqq 1-b^{2}+\frac{1}{4} v^{2}-\frac{1}{4} \mu^{2} \frac{\log b}{1+\log b} .
$$

Equality is possible if and only if

$$
\begin{array}{ll}
\text { i) } & \operatorname{Im} a_{2}+\frac{1}{2} v=0, \\
\text { ii) } & \operatorname{Re} a_{2}+\frac{\mu \log b}{2(1+\log b)}=0, \\
\text { iii) } & \operatorname{Re} A(u)=0 \text { i.e. } \cos \vartheta(u)=-\frac{\operatorname{Re} a_{2}+\mu / 2}{2 u} .
\end{array}
$$

We shall show that (5) is sharp for some numbers $\lambda=\mu+i$. 
Let us choose $|\mu| \leqq 4 b(1+\log b)$ and let

$$
\sigma=\frac{|\mu|}{4(1+\log b)}
$$

therefore $0 \leqq \sigma \leqq b$. Define $\vartheta$ in such a way that

$$
\begin{aligned}
& \cos \vartheta(u)=\frac{\sigma}{u}, \\
& \sin \vartheta(u)=\left\{\begin{array}{l}
\sqrt{1-\frac{\sigma^{2}}{u^{2}}} \text { for } \quad b \leqq u \leqq c, \\
-\sqrt{1-\frac{\sigma^{2}}{u^{2}}} \text { for } c<u \leqq 1 .
\end{array}\right.
\end{aligned}
$$

The point $c$ will be chosen later. For this $\vartheta$ we have

$$
\operatorname{Re} a_{2}=-2 \int_{b}^{1} \cos \vartheta(u) d u=\frac{|\mu| \log b}{2(1+\log b)}=-\frac{\mu \log b}{2(1+\log b)}
$$

thus ii) is satisfied. Now it follows that

$$
\operatorname{Re} a_{2}+\frac{1}{2} \mu=\frac{\mu}{2(1+\log b)}=-2 \sigma
$$

which means that iii) holds. In order to show i) we choose $c$ such that $\operatorname{Im} a_{2}=$ $-2 \int_{b}^{1} \sin \vartheta(u) d u=-v / 2$. This is possible so far as

$$
\frac{1}{2}|v| \leqq 2 \int_{b}^{1}|\sin \vartheta(u)| d u
$$

i.e. $|v| \leqq 4\left(\sqrt{1-\sigma^{2}}-\sqrt{b^{2}-\sigma^{2}}+\sigma \overline{\operatorname{arc}} \cos \sigma / b-\sigma \overline{\operatorname{arc}} \cos \sigma\right)$.

The equality case for $\mu>0$ can be handled similarly. Collecting the results we arrive at

Theorem 1. Let $e^{-1}<b<1, \lambda=\mu+i v, \sigma=|\mu| / 4(1+\log b)$. If

$$
\left\{\begin{array}{l}
|\mu| \leqq 4 b(1+\log b), \\
|v| \leqq 4\left(\sqrt{1-\sigma^{2}}-\sqrt{b^{2}-\sigma^{2}}+\sigma \overline{\operatorname{arc}} \cos \frac{\sigma}{b}-\sigma \overline{\operatorname{arc} c o s} \sigma\right)
\end{array}\right.
$$

then

$$
\max _{f \in S(b)} \operatorname{Re}\left(a_{3}+\lambda a_{2}\right)=1-b^{2}+\frac{1}{4} v^{2}-\frac{1}{4} \mu^{2} \frac{\log b}{1+\log b} .
$$

The maximum is reached for a function mapping $\Delta$ onto $\Delta$ minus two slits. 
Note. If $b=e^{-1}$, then similar arguments show that to each $v$ with $|v|<4\left(1-e^{-1}\right)$ there belongs a one-parametric family of extremal functions parametrized by $\operatorname{Re} a_{2} \in\left[-2 e^{-1}, 2 e^{-1}\right]$.

\section{Extremals of type $1: 2$}

Now we take (4b) as a starting point. Let $|\mu| \leqq 4 b, t=-\left(\operatorname{Re} a_{2}+\mu / 2\right) / 2$. From $\left|a_{2}\right| \leqq 2(1-b)$ it follows that $0 \leqq t \leqq 1$. In this notation we have

$$
\operatorname{Re} A(u)=\sqrt{u}\left(\cos \vartheta(u)-\frac{t}{u}\right) .
$$

For all functions $\vartheta$ the following holds. If $t \leqq b$, we have the trivial estimate $|\cos \vartheta(u)-t / u| \geqq 0$ for $b \leqq u \leqq 1$. If $t>b$, we can say more:

Therefore, we have

$$
\left|\cos \vartheta(u)-\frac{t}{u}\right| \geqq \begin{cases}\left|1-\frac{t}{u}\right| & \text { for } \quad b \leqq u \leqq t \\ 0 & \text { for } t \leqq u \leqq 1 .\end{cases}
$$

and thus

$$
-(\operatorname{Re} A(u))^{2} \geqq \begin{cases}-u\left(1-\frac{t}{u}\right)^{2} & \text { for } \quad b \leqq u \leqq t \\ 0 & \text { for } t \leqq u \leqq 1,\end{cases}
$$

$$
-4 \int_{b}^{1}(\operatorname{Re} A(u))^{2} d u \leqq 6 t^{2}-4 t^{2} \log t+2 b^{2}-8 t b+4 t^{2} \log b,
$$

with the equality if and only if

From (4b) we obtain now

$$
\cos \vartheta(u)= \begin{cases}1 & \text { for } \quad b \leqq u \leqq t \\ \frac{t}{u} & \text { for } t \leqq u \leqq 1\end{cases}
$$

where

$$
\operatorname{Re}\left(a_{3}+\lambda a_{2}\right) \leqq g(t)
$$

$$
g(t)=\left\{\begin{array}{rr}
1-b^{2}+\frac{1}{4} v^{2}-\frac{1}{4} \mu^{2}-4 t^{2}-2 t \mu-4 t^{2} \log b-\left(\operatorname{Im} a_{2}+\frac{1}{2} v\right)^{2} & \text { for } 0 \leqq t \leqq b, \\
1-b^{2}+\frac{1}{4} v^{2}-\frac{1}{4} \mu^{2}-4 t^{2}-2 t \mu+6 t^{2}-4 t^{2} \log t+2 b^{2}-8 t b-\left(\operatorname{Im} a_{2}+\frac{1}{2} v\right)^{2} & \text { for } b \leqq t \leqq 1 .
\end{array}\right.
$$

This function $g$ is differentiable on $[0,1]$ and

$$
g^{\prime}(t)=\left\{\begin{array}{lll}
-8 t-2 \mu-8 t \log b & \text { for } & 0 \leqq t \leqq b, \\
-2 \mu-8 t \log t-8 b & \text { for } & b \leqq t \leqq 1 .
\end{array}\right.
$$

Consider first the case $e^{-1}<b<1$ and take

$$
4 b(1+\log b) \leqq|\mu| \leqq 4 b .
$$


Now $g^{\prime}(t) \geqq 0$ on $[0, b)$ and hence $g$ has its maximum on $[b, 1]$, where $g^{\prime}$ has one zero $\sigma$. This $\sigma$ is determined by the condition. $-4 \sigma \log \sigma=4 b+\mu$. We obtain

$$
\max _{0 \leqq t \leqq 1} g(t)=g(\sigma)=1+b^{2}+\frac{1}{4} v^{2}-\frac{1}{4} \mu^{2}+2 \sigma^{2}-\sigma \mu-4 \sigma b
$$

and thus

$$
\operatorname{Re}\left(a_{3}+\lambda a_{2}\right) \leqq 1+b^{2}+\frac{1}{4} \nu^{2}-\frac{1}{4} \mu^{2}+2 \sigma^{2}-\sigma \mu-4 \sigma b,
$$

where the equality occurs if and only if

i)

$$
\begin{aligned}
& \cos \vartheta(u)=\left\{\begin{array}{lll}
1 & \text { for } & b \leqq u \leqq \sigma, \\
\frac{\sigma}{u} & \text { for } & \sigma \leqq u \leqq 1,
\end{array}\right. \\
& \sigma=-\frac{1}{2}\left(\operatorname{Re} a_{2}+\frac{1}{2} \mu\right) \\
& -4 \sigma \log \sigma=4 b+\mu \text {, }
\end{aligned}
$$

ii)

iv)

$$
\operatorname{Im} a_{2}+\frac{1}{2} v=0 .
$$

In order to show that these conditions can be satisfied simultaneously we consider $\mu$ with

$$
-4 b \leqq \mu \leqq-4 b(1+\log b) .
$$

There is one $\sigma \geqq e^{-1}$ with $-4 \sigma \log \sigma=4 b+\mu$. Define

$$
\cos \vartheta(u)=\left\{\begin{array}{lll}
1 & \text { for } \quad b \leqq u \leqq \sigma, \\
\frac{\sigma}{u} & \text { for } \quad \sigma \leqq u \leqq 1 .
\end{array}\right.
$$

So far the conditions i), ii) and iii) are satisfied. In order to make iv) hold we have to require

$$
\frac{1}{4}|v| \leqq \int_{b}^{1}|\sin \vartheta(u)| d u .
$$

The equality case $\mu>0$ is treated similarly. The results collected give

Theorem 2. Let $e^{-1}<b<1, \lambda=\mu+i v$ and $\sigma \in\left[e^{-1}, 1\right]$ be determined by

If

$$
-4 \sigma \log \sigma=4 b-|\mu| \text {. }
$$

we have

$$
\left\{\begin{array}{l}
4 b(1+\log b) \leqq|\mu| \leqq 4 b, \\
|v| \leqq 4\left(\sqrt{1-\sigma^{2}}-\sigma \overline{\operatorname{arc} c o s} \sigma\right),
\end{array}\right.
$$

$$
\max _{f \in S(b)} \operatorname{Re}\left(a_{3}+\lambda a_{2}\right)=1+b^{2}+\frac{1}{4} v^{2}-\frac{1}{4} \mu^{2}+2 \sigma^{2}+\sigma|\mu|-4 \sigma b .
$$

The maximum is reached for a function mapping $\Delta$ onto $\Delta$ minus a forked slit. 
Next consider the case $0<b \leqq e^{-1}$. From (6) we see that $g^{\prime}(t) \geqq 0$ on $[0, b]$. Thus, again, $g$ has its maximum on $[b, 1]$. Arguments similar to those in the previous case lead to

Theorem 3. Let $0<b \leqq e^{-1}, \lambda=\mu+i v$ and $\sigma \in\left[e^{-1}, 1\right]$ is determined by

If

$$
-4 \sigma \log \sigma=4 b-|\mu| \text {. }
$$

we have

$$
\left\{\begin{array}{l}
|\mu| \leqq 4 b, \\
|v| \leqq 4\left(\sqrt{1-\sigma^{2}}-\sigma \overline{\operatorname{arc}} \cos \sigma\right)
\end{array}\right.
$$

$$
\max _{f \in S(b)} \operatorname{Re}\left(a_{3}+\lambda a_{2}\right)=1+b^{2}+\frac{1}{4} v^{2}-\frac{1}{4} \mu^{2}+2 \sigma^{2}+\sigma|\mu|-4 \sigma b .
$$

The maximum is reached for a function mapping $\Delta$ onto $\Delta$ minus a forked slit.

\section{Extremals of type 1:1}

A particular case of extremals of type 1:1 is obtained if $\mu=0$. From (3) we see that for such an extremal we have

$\sin \vartheta(u) \int_{b}^{1} \cos \vartheta(u) d u+\cos \vartheta(u) \int_{b}^{1} \sin \vartheta(u) d u-u \sin \vartheta(u) \cos \vartheta(u)=\frac{1}{4} v \int_{b}^{1} \cos \vartheta(u) d u$.

Integration over $[b, 1]$ gives

$$
2 \int_{b}^{1} \sin \vartheta(u) d u \int_{b}^{1} \cos \vartheta(u) d u-\int_{b}^{1} u \sin \vartheta(u) \cos \vartheta(u) d u=\frac{1}{4} v \int_{b}^{1} \cos \vartheta(u) d u .
$$

We consider only those cases where $v \geqq 4(1-b)$. We know that in the maximum case $\sin \vartheta(u) \geqq 0$ and thus

$$
v \int_{b}^{1} \cos \vartheta(u) d u \leqq 8 \int_{b}^{1} \sin \vartheta(u) d u \int_{b}^{1} \cos \vartheta(u) d u \leqq 8(1-b) \int_{b}^{1} \cos \vartheta(u) d u .
$$

Therefore, if $\int_{b}^{1} \cos \vartheta(u) d u \neq 0$, we must have $v \leqq 8(1-b)$. It is clear that we have even $v<8(1-b)$.

Theorem 4. For $0<b<1$ assume that $|v| \geqq 8(1-b)$. Then

$$
\max _{f \in S(b)} \operatorname{Re}\left(a_{3}+i v a_{2}\right)=-3+8 b-5 b^{2}+2(1-b)|v| \text {. }
$$

The maximum is reached for a function $f$ for which

$$
b\left(f-\frac{1}{f}\right)=z-\frac{1}{z} \pm 2(1-b) i .
$$

This function maps $\Delta$ onto $\Delta$ minus a rectilinear slit. 
If $e^{-1} \leqq b<1$, we can say more. From (4) we see that

$$
\operatorname{Re}\left(a_{3}+i v a_{2}\right) \leqq 1-b^{2}+\frac{1}{4} v^{2}-\left(\operatorname{Im} a_{2}+\frac{1}{2} v\right)^{2} .
$$

If $y \geqq 4(1-b)$, it follows from $\left|a_{2}\right| \leqq 2(1-b)$ that

$$
\begin{gathered}
\left|\operatorname{Im} a_{2}+\frac{1}{2} v\right| \geqq \frac{1}{2} v-2(1-b) ; \\
\operatorname{Re}\left(a_{3}+i v a_{2}\right) \leqq-3+8 b-5 b^{2}+2(1-b) v .
\end{gathered}
$$

The equality sign holds if $\sin \vartheta(u) \equiv 1$. Negative values of $v$ can be treated similarly.

Theorem 5. For $e^{-1} \leqq b<1$ assume that $|v| \geqq 4(1-b)$. Then

$$
\max _{f \in S(b)} \operatorname{Re}\left(a_{3}+i v a_{2}\right)=-3+8 b-5 b^{2}+2(1-b)|v| .
$$

The maximum is reached for a function $f$ for which

$$
b\left(f-\frac{1}{f}\right)=z-\frac{1}{z} \pm 2(1-b) i .
$$

This function maps $\Delta$ onto $\Delta$ minus a rectilinear slit.

The general cases $1: 1$ remain to be discussed. Let

$$
p=\int_{b}^{1} \cos \vartheta(u) d u-\frac{1}{4} \mu \geqq 0, \quad q=\int_{b}^{1} \sin \vartheta(u) d u-\frac{1}{4} v .
$$

The variational formula (3) thus assumes the form

$$
p \sin \vartheta(u)+q \cos \vartheta(u)=u \sin \vartheta(u) \cos \vartheta(u) .
$$

We have to consider four alternatives with respect to $\sin \vartheta(u)$ and $\cos \vartheta(u)$.

$1^{\circ}$ There exists a value $u$ for which $\sin \vartheta(u)=0$.

From (7) if follows that $q=0$ and hence $v \leqq 4(1-b)$. We can say even more. Because $|\sin \vartheta|$ is increasing, there exists a number $c \in[b, 1]$ such that $\sin \vartheta(u)=0$ on $[b, c), \sin \vartheta(u) \neq 0$ on $(c, 1]$. Therefore we see from (7) that

$$
\cos \vartheta(u)=\left\{\begin{array}{lll}
1 & \text { on } \quad[b, c), \\
\frac{p}{u} & \text { on } \quad(c, 1] ; c \geqq p .
\end{array}\right.
$$

From $q=0$ it follows further that

$$
\frac{1}{4} v \leqq \int_{b}^{1}|\sin \vartheta(u)| d u=\int_{c}^{1} \sqrt{1-p^{2} / u^{2}} d u \leqq \int_{p}^{1} \sqrt{1-p^{2} / u^{2}} d u=\sqrt{1-p^{2}}-p \overline{\operatorname{arc}} \cos p
$$

thus

$$
v \leqq 4\left(\sqrt{1-p^{2}}-p \overline{\operatorname{arc}} \cos p\right) .
$$


By using (2) we decide that for a prescribed $p, \operatorname{Re}\left(a_{3}+\lambda a_{2}\right)$ is maximal if

$$
\int_{b}^{1} u \cos ^{2} \vartheta(u) d u=\frac{1}{2}\left(c^{2}-b^{2}\right)-p^{2} \log c
$$

is minimal, i.e. if $c=p$. Thus the maximizing choice of $c$ and $\cos \vartheta(u)$ is

which gives

$$
\cos \vartheta(u)=\left\{\begin{array}{lll}
1 & \text { on } & {[b, p],} \\
\frac{p}{u} & \text { on } & {[p, 1]}
\end{array}\right.
$$

$$
p=\int_{b}^{1} \cos \vartheta(u) d u-\frac{1}{4} \mu=p-b-p \log p-\frac{1}{4} \mu,
$$

i.e.

$$
-4 p \log p=4 b+\mu .
$$

If $e^{-1}<b<1$, the previous condition implies, because $p \in[b, 1]$, that

$$
4 b(1+\log b) \leqq|\mu| \leqq 4 b .
$$

Similarly, if $0<b \leqq e^{-1}$, we obtain

$$
|\mu| \leqq 4 b .
$$

Therefore, in the case $1^{\circ} \operatorname{Re}\left(a_{3}+\lambda a_{2}\right)$ is maximized, according to Theorems 1 and 3 , by extremal functions of the type $1: 1$.

Next, consider the remaining cases where

$2^{\circ} \sin \vartheta(u) \neq 0$.

The following alternatives are to be checked.

1) $p=0$.

$$
0 \geqq \frac{1}{4} \mu=\int_{b}^{1} \cos \vartheta(u) d u \geqq 0 .
$$

Thus

$$
\mu=0, \quad \cos \vartheta(u) \equiv 0 \quad \text { and } \quad|\sin \vartheta(u)| \equiv 1 .
$$

There are two possibilities available.

If $v \geqq 4(1-b)$, we know that in the maximum case $\sin \vartheta(u) \geqq 0$, i.e. $\sin \vartheta(u) \equiv 1$, and therefore we are led to the cases of Theorem 5, where

$$
\operatorname{Re}\left(a_{3}+i v a_{2}\right)=-3+8 b-5 b^{2}+2 v(1-b) .
$$

If $v<4(1-b)$, we see from (2) that

$$
\operatorname{Re}\left(a_{3}+\lambda a_{2}\right)=1-b^{2}+\frac{1}{4} v^{2}-\left(\operatorname{Im} a_{2}+\frac{v}{2}\right)^{2} \leqq 1-b^{2}+\frac{1}{4} v^{2},
$$


where the equality, making $\operatorname{Im} a_{2}=-v / 2$, is reached for

$$
\sin \vartheta(u)=\left\{\begin{array}{rrr}
1 & \text { on } & {\left[b, \frac{1+b}{2}+\frac{v}{8}\right],} \\
-1 & \text { on } & \left(\frac{1+b}{2}+\frac{v}{8}, 1\right] .
\end{array}\right.
$$

This maximum thus belongs to the cases of Theorem 1 .

2) $p>0, q=0$.

From (7) we see that $\cos \vartheta(u)=p / u, p \leqq b$ and $|\sin \vartheta(u)|=\sqrt{1-p^{2} / u^{2}}$. Further,

$$
\begin{aligned}
\frac{1}{4} v & \leqq \int_{b}^{1}|\sin \vartheta(u)| d u=\int_{b}^{1} \sqrt{1-p^{2} / u^{2}} d u \\
& =\sqrt{1-p^{2}}-\sqrt{b^{2}-p^{2}}+p \overline{\operatorname{arc} c o s} \frac{p}{b}-p \overline{\operatorname{arc} c o s} p .
\end{aligned}
$$

Because $p=\int_{b}^{1} \cos \vartheta(u) d u-\mu / 4$, we have

$$
p+\frac{1}{4} \mu=-p \log b ; \quad p=\frac{-\mu}{4(1+\log p)} .
$$

From $p>0$ it follows now that $b \in\left(e^{-1}, 1\right)$, and therefore we are in the cases of Theorem 1 .

3) $p>0, q>0$.

Now we have $v / 4<\int_{b}^{1} \sin \vartheta(u) d u \leqq 1-b$. Here one can repeat the conclusions on pp. 132-134, i.e. changing the signs of $\vartheta$ propely without affecting $\cos \vartheta$ we can always diminish $\int_{b}^{1} \sin \vartheta(u) d u$ into the value $v / 4$. This new $\vartheta$ increases $\operatorname{Re}\left(a_{3}+\lambda a_{2}\right)$ to its maximum. Because for the new $\vartheta q=0$, we see that 3 ) is not the maximum case.

$3^{\circ} \sin \vartheta(u)$ obtains negative values.

According to the remark on p. 134 we know that in the maximum case necessarily $\int_{b}^{1} \sin \vartheta(u) d u=v / 4$, i.e. $q=0$. From (7) we see that we can now go back to the function $\vartheta$ which was defined in $1^{\circ}$ and thus we end up with the same conclusions as in $1^{\circ}$.

$4^{\circ}$ There exists a value $u$ for which $\cos \vartheta(u)=0$.

In this case $p=0$, but because $\cos \vartheta(u) \geqq 0$ and $\mu \leqq 0$, we must have $\cos \vartheta(u) \equiv 0$ and $\mu=0$. We are led back to the beginning of $2^{\circ}$, where this case was handled under the assumption $p=0$.

From $1^{\circ}-4^{\circ}$ we decide now that in the cases not handled yet there are

$$
p>0, \quad q<0, \quad \cos \vartheta>0, \quad \sin \vartheta>0 .
$$

Rewrite (7) in the form

$$
F(\vartheta, u)=\frac{p}{\cos \vartheta}+\frac{q}{\sin \vartheta}-u=0 .
$$


Because $F_{\vartheta}(\vartheta, u)>0$ we see that $(7 \mathrm{a})$ determines $\vartheta$ as a differentiable function of $u$ and

$$
d u=\left(\frac{p \sin \vartheta}{\cos ^{2} \vartheta}-\frac{q \sin \vartheta}{\sin ^{2} \vartheta}\right) d \vartheta .
$$

It follows from the Löwner theory [3] that such a function $\vartheta$ determines a solution of type $1: 1$.

If we denote

$$
\alpha=-\vartheta(1), \quad \omega=-\vartheta(b),
$$

we obtain from (3) the equations

$$
\left\{\begin{array}{l}
p \sin \alpha-q \cos \alpha=\sin \alpha \cos \alpha \\
p \sin \omega-q \cos \omega=b \sin \omega \cos \omega .
\end{array}\right.
$$

Two more equations can be obtained from

$$
\left\{\begin{aligned}
p=\int_{b}^{1} \cos \vartheta(u) d u-\frac{1}{4} \mu & =\int_{-\omega}^{-\alpha} \cos \vartheta\left(\frac{p \sin \vartheta}{\cos ^{2} \vartheta}-\frac{q \cos \vartheta}{\sin ^{2} \vartheta}\right) d \vartheta-\frac{1}{4} \mu \\
& =-p \log \frac{\cos \alpha}{\cos \omega}-q(\cot \alpha-\cot \omega+\alpha-\omega)-\frac{\mu}{4} \\
q=\int_{b}^{1} \sin \vartheta(u) d u-\frac{1}{4} v & =-p(\tan \alpha-\tan \omega-\alpha+\omega)-q \log \frac{\sin \alpha}{\sin \omega}-\frac{v}{4}
\end{aligned}\right.
$$

From (2) we see that

$$
\begin{gathered}
\max _{f \in S(b)} \operatorname{Re}\left(a_{3}+\lambda a_{2}\right)=1-b^{2}-\frac{1}{4} \mu^{2}+\frac{1}{4} \nu^{2}-p \mu+q \nu+4 p q(\tan \alpha-\tan \omega) \\
-2 q^{2}\left(\frac{1}{\sin ^{2} \alpha}-\frac{1}{\sin ^{2} \omega}\right) .
\end{gathered}
$$

For the original problem $\max _{f \in S} \operatorname{Re}\left(a_{3}+i a_{2}\right)$ we have determined the follow. ing numerical solution:

$$
\begin{gathered}
\left\{\begin{array}{l}
\alpha=-0.528 \cdot 513 \cdot 532 \\
\omega=-0.066 \cdot 344 \cdot 080
\end{array}\right. \\
\max _{f \in S} \operatorname{Re}\left(a_{3}+i a_{2}\right)=3.190 \cdot 298 \cdot 109 .
\end{gathered}
$$

Note 1 . From (3a) it follows that $\vartheta \equiv \pi / 2$ gives at least a local maximum if and only if

$$
v \geqq 4(1-b)+4 \frac{\left(\int_{b}^{1} \varphi(u) d u\right)^{2}-\int_{b}^{1} u \varphi^{2}(u) d u}{\int_{b}^{1} \varphi^{2}(u) d u}
$$


for all piecewise continuous functions $\varphi$ on $[b, 1]$. If $b \in\left[e^{-1}, 1\right)$, we have from Schwarz's inequality

$$
\left(\int_{b}^{1} \varphi(u) d u\right)^{2} \leqq \int_{b}^{1} \frac{d u}{u} \int_{b}^{1} u \varphi^{2}(u) d u=-\log b \int_{b}^{1} u \varphi^{2}(u) d u \leqq \int_{b}^{1} u \varphi^{2}(u) d u .
$$

The condition for $v$ is thus in accordance with Theorem 5.

Note 2. By solving the system (8) with the aid of power series in the neighbourhood of $\alpha=\omega=\pi / 2$ we find that for $|v| \geqq 4(1-b)+4(1-e b) /(e-1)$ the functions with one rectilinear slit give $\max _{f \in S(b)} \operatorname{Re}\left(a_{3}+i v a_{2}\right)$.

Note 3. The problem of determining $\min _{f \in S(b)} \operatorname{Re}\left(a_{3}+\lambda a_{2}\right)$ is easily reduced to the problem studied here. By considering -if(iz) instead of $f(z)$ we see that

$$
\min _{f \in S(b)} \operatorname{Re}\left(a_{3}+\lambda a_{2}\right)=-\max _{f \in S(b)} \operatorname{Re}\left(a_{3}+i \lambda a_{2}\right) .
$$

Note 4. By using the same arguments as before one can also determine the part of the coefficient body $\left(a_{2}, a_{3}\right)$ of $S(b)$ where the boundary functions are of the type $1: 1$.

\section{References}

[1] HaARIo, H., and O. Jokinen: On the use of Löwner's functions for finding inequalities for the first coefficient body of bounded univalent functions. - Reports of the Department of Mathematics, Ser. A No. 14, 1977, University of Helsinki.

[2] Kortram, R., and O. TAMMI: On the first coefficient regions of bounded univalent functions. Ann. Acad. Sci. Fenn. Ser. A I 592, 1974, 1-27.

[3] Ку фарев, П.П.: Об интегралах простейшего дифференциального уравнения с подвижной полярной особенностью правой части. -Tomsk. Gos. Univ. Učen. Zap. No. $1,1946,35-48$.

[4] Schober, G.: Univalent functions - Selected topics. - Lecture Notes in Mathematics 478, Springer-Verlag, Berlin-Heidelberg-New York, 1975.

[5] TAмmi, O.: On the conformal mapping of symmetric schlicht domains. - Ann. Acad. Sci. Fenn. Ser. A I 173, 1954, 1-12.

University of Nijmegen

Department of Mathematics

Nijmegen

The Netherlands

Received 1 August 1979
University of Helsinki

Department of Mathematics

SF-00100 Helsinki 10

Finland 